\title{
Potassium Carbonate as a Salt for Deep Eutectic Solvents
}

\author{
J. Naser, F. Mjalli, B. Jibril, S. Al-Hatmi, and Z. Gano
}

\begin{abstract}
Deep Eutectic Solvents (DES) are emerging as new class of green solvents with the favorable properties of low cost, minimum volatility, biodegradability and suitability for many industrial applications. In an attempt to synthesize a new class of DES, potassium carbonate was used as a salt with glycerol as a hydrogen bond donor (HDB). The basic physical properties of this DES were measured at different temperatures and salt:HBD molar ratios. The physical properties measured included density, viscosity, surface tension, refractive index and $\mathrm{pH}$ at a temperature range of $20-80^{\circ} \mathrm{C}$. The system didn't show a freezing points in DSC thermograms, however glass transition temperatures were observed. This system exhibits similar physico-chemical properties to other reported DES. The values of these properties indicated that the prepared DES may have great potential for industrial applications.
\end{abstract}

Index Terms-Potassium carbonate, glycerol, deep eutectic solvents, ionic liquids, properties.

\section{INTRODUCTION}

Room temperature ionic liquids (ILs) are gaining increasing interest by both scientific and industrial communities. These are relatively new class of solvents which are poised to solve both the economic and environmental challenges.

Ionic liquids individual physical and chemical properties can vary over a broad range. However, the attracting room temperature properties of these liquids make them suitable for many industrial applications. Due to their ionic nature and large molar mass, ILs attain low vapor pressures [1]-[2]. The low volatility allows greener synthesis with reduced environmental impacts, in contrast to volatile organic solvents [3]. Their low molar densities make their physicochemical properties especially sensitive to impurities or additives. In addition ILs have wide liquid temperature range, high selectivity and solubility for many organic or inorganic compounds, and less toxicity [4]-[6]. ILs are immiscible with many organic solvents; moreover, the solvation properties of ILs can be tuned for a specific application by varying the anion-cation combinations [7]

Such liquids have a great potential for capturing different gases with minimal solvent loss in the gas stream due to their high selectivity. $\mathrm{CO}_{2}$ capture is one related application that has shown great achievements with superior uptake compared to conventional absorbents [8].

A major obstacle against the wide spread use of ILs in the industry is their high synthesis cost compared to conventional solvents. A green alternative for traditional ILs would be composed of biodegradable constituents and exhibit high

Manuscript received February 11, 2013; revised April 12, 2013.

The authors are with Department of Petroleum and Chemical Engineering, Sultan Qaboos University, Sultanate of Oman (e-mail: naserj@ squ.edu.om). thermal and chemical stabilities. Deep eutectic solvents are one of such alternatives. These are simple mixtures of salts and hydrogen bond donor compounds such as alcohols, acids, halides, amines, amino acids and many more. Deep eutectic solvents (DESs) are regarded as ionic liquids analogues because they share many of their intrinsic favorable properties such as their bio-degradability, non-flammability due to their low or none measurable vapor pressure and low toxicity [1], [2] and [9]. Moreover, the fact that they are synthesized at conditions close to room temperature reduces their cost and makes them readily available for probable industrial scales.

Conventionally, deep eutectic solvents have been synthesized from ammonium or phosphonium based salts. These salts were combined in different ratios with varieties of hydrogen bond donors such as: alcohols, carboxylic acids, esters, ethers, amides, and hydrated metal salts of chlorides, nitrates and acetates [10]. In addition, metal halides based DESs were also reported later [11]. However, potassium based salts were not used an ingredient for DES synthesis. Potassium carbonate $\left(\mathrm{K}_{2} \mathrm{CO}_{3}\right)$ known as pearl ash is a white salt having a melting point of $1164.15 \mathrm{~K}$. It is highly soluble in water $(112 \mathrm{~g} / 100 \mathrm{~mL}$ at $293.15 \mathrm{~K})$ forming a strong alkaline solution.

In its aqueous form, potassium carbonate is utilized for the removal of carbon dioxide from the ammonia production synthesis gas in the fertilizers industry [12].

Due to its low heat capacity, $\mathrm{K}_{2} \mathrm{CO}_{3}$ solutions are also used as promoters for primary or secondary amines for $\mathrm{CO}_{2}$ capture. It was also used in combinations with the diamine piperazine for enhancing $\mathrm{CO}_{2}$ absorption. In this case potassium carbonate in solution provides an additional sink for "storage" of the absorbed $\mathrm{CO}_{2}[13]$.

Glycerol (1, 2, 3-propanetriol) is a non-toxic, colorless, odorless, viscous liquid with a sweet taste, derived from both natural and petrochemical feedstocks. The name glycerol is derived from the Greek word for sweet (glykys), and the terms glycerin, glycerine, and glycerol tend to be used interchangeably in the literature [14]. In its pure form, Glycerol has 1.261 specific gravity, $1500 \mathrm{cP}$ viscosity, 64 dyne/cm surface tension (all at $293.15 \mathrm{~K}$ ) and a freezing point of 18.2 C. In Deep Eutectic Solvents, Glycerol has been successfully used as a hydrogen bond donor with Choline Chloride to form DES by several research groups [15]-[16].

In this study, potassium carbonate and glycerol were used in different molar ratios to make a new DES system. The important physical properties of density, viscosity, surface tension, refractive index, and $\mathrm{pH}$ were investigated at different temperatures. The rationale of studying such systems arises from the need of developing a green solvent that can be utilized economically for possible applications including but not limited to gas absorption, liquid-liquid extraction and catalysis. To explore the possibility of such 
application, the physical properties of such new classes of DESs need to be evaluated and predicted.

\section{EXPERIMENTAL METHODOLOGY}

\section{A. Chemicals Used}

Potassium carbonate and glycerol (> $98 \%$ ), were supplied by Merck Chemicals (Darmstadt, Germany). These chemicals were used without any further purification.

\section{B. Preparation the DESS}

DESs samples were synthesized in different molar ratios of potassium carbonate to glycerol. An incubator shaker (Brunswick Scientific Model INNOVA 40R) was used to mix the salt and the hydrogen bond donor. Each DES mixture was shaken at $400 \mathrm{rpm}$ and $353.15 \mathrm{~K}$ for a period of 2 hours until a homogenous transparent colorless liquid was formed. DES samples were synthesized at atmospheric pressure and under tight control of moisture content.

\section{Physical Properties Measurement}

Prepared samples were kept in airtight sealed glass vials after preparation and fresh samples were used for analysis to avoid any structural change during storage and to avoid humidity effects from the environment which may affect the physical properties of DES.

DES samples were dried under vacuum overnight in order to eliminate any possible contamination with moisture before measuring the density. The densities were measured using Anton Paar DMA4500M while the viscosities of the DESs were measured using Anton Paar Rheolab Qc. The temperature was controlled using external water-circulator type Techne-Tempette TE-8A. The surface tensions were measured using an automated tensiometer Krüss K10ST classification B with Du Noüy ring method. Refractive indices were measured using a Bellingham and Stanley Abbe Refractometer (model 60/ED) with a sodium D1 line. The temperature was controlled in the refractometer using Techno TE-8D water circulator. Deionized water was used for calibration before each experiment. The $\mathrm{pH}$ of samples were measured using Thermo Scientific 3 star $\mathrm{pH}$ Bench top meter. The $\mathrm{pH}$ meter was calibrated using a standard $\mathrm{pH}$ buffer. The temperature of each sample was controlled using a water circulator (Julabo Labortechnik).

\section{RESULTS AND DISCUSSION}

Different molar ratios of glycerol to potassium carbonate were used to prepare the DESs. Table I shows these ratios along with their abbreviations and observations during the preparation process.

The different DES ratios were prepared by varying glycerol molar ratio at a fixed amount of salt. DES1, DES2, and DES3 were not successful as the two components did not form DES and the products were in either turbid white liquid or a mixture of colorless liquid and solid throughout the process and after cooling to room temperature. The presence of solid particles in the mixture indicates that the amount of salt is in excess to the corresponding hydrogen bond donor which results in the precipitation of the extra amount of salt which cannot build hydrogen bonds with the hydrogen bond donor.

TABLE I: Molar RATIOS AND ABBREVIATIONS OF DESS PREPARED

\begin{tabular}{lcl}
\hline Ratio & Abbreviation & Appearance \\
\hline $1: 1$ & DES1 & turbid white liquid \\
$1: 2$ & DES2 & colorless liquid with some solid \\
$1: 3$ & DES3 & colorless liquid with little solid \\
$1: 3.5$ & DES4 & colorless liquid \\
$1: 4$ & DES5 & colorless liquid \\
$1: 5$ & DES6 & colorless liquid \\
$1: 6$ & DES7 & colorless liquid \\
$1: 10$ & DES8 & colorless liquid \\
$1: 20$ & DES9 & colorless liquid \\
$1: 50$ & DES10 & colorless liquid \\
$1: 75$ & DES11 & colorless liquid \\
$1: 100$ & DES12 & colorless liquid \\
\hline
\end{tabular}

During the synthesis stage, DESs samples were formed in a white viscous gel within the first $30 \mathrm{~min}$. After $60 \mathrm{~min}$ of mixing, a liquid phase started to appear with some precipitation. Therefore, the period of mixing was extended to $120 \mathrm{~min}$ in order to get a homogenous liquid phase DES. Adding more glycerol achieved the necessary balance between the two DES constituents and guaranteed complete miscibility.

The three unsuccessful DESs were not considered for further investigation in this study. On the other hand, DES4-DES12 remained in colorless liquid phase at room temperature.

Differential Scanning Calorimeter (DSC ) model number TA Q1000 was used for measuring the freezing points of the synthesized DESs. The DSC was calibrated using Indium DSC standard and the modulated DSC mode was used for the measurements.

Three ratios, 1:4, 1:5 and 1:6 were selected for measuring the physical properties (density, viscosity, surface tension, conductivity, refractive index, and $\mathrm{pH}$ ). All physical properties were measured at $10{ }^{\circ} \mathrm{C}, 20{ }^{\circ} \mathrm{C}, 30{ }^{\circ} \mathrm{C}, 40{ }^{\circ} \mathrm{C}, 50$ ${ }^{\circ} \mathrm{C}, 60{ }^{\circ} \mathrm{C}, 70^{\circ} \mathrm{C}$ and $80^{\circ} \mathrm{C}$.

Potassium carbonate has a freezing point of $891^{\circ} \mathrm{C}$ while that of glycerol is $17^{\circ} \mathrm{C}$. All the analyzed DESs didn't show a freezing point in the DSC thermogram, instead a glass transition temperature was observed in all of them. DES4 had a glass transition temperature of $-38^{\circ} \mathrm{C}$ and as the molar ratio glycerol increased the glass transition temperature decreased to $-78.54{ }^{\circ} \mathrm{C}$ at $1: 50$ and almost remained unchanged for the rest of the ratios. This temperature drop is shown Fig. 1 for all DESs ratios studied in this work.

As can be seen from Fig. 1, the temperatures continued dropping to reach an almost stable temperature of $-77.5^{\circ} \mathrm{C}$ for the last three ratios. The DSC thermograms did show a freezing point peak for all ratios. The most evident event in the thermogram was the glass transition slope that was used to estimate the glass transition temperature for the system. This phenomenon was recently observed by [17] using previously reported DES system of Choline Chloride-lactic acid in which glass transition was observed rather than freezing for a wide range of ratios used in their work. 


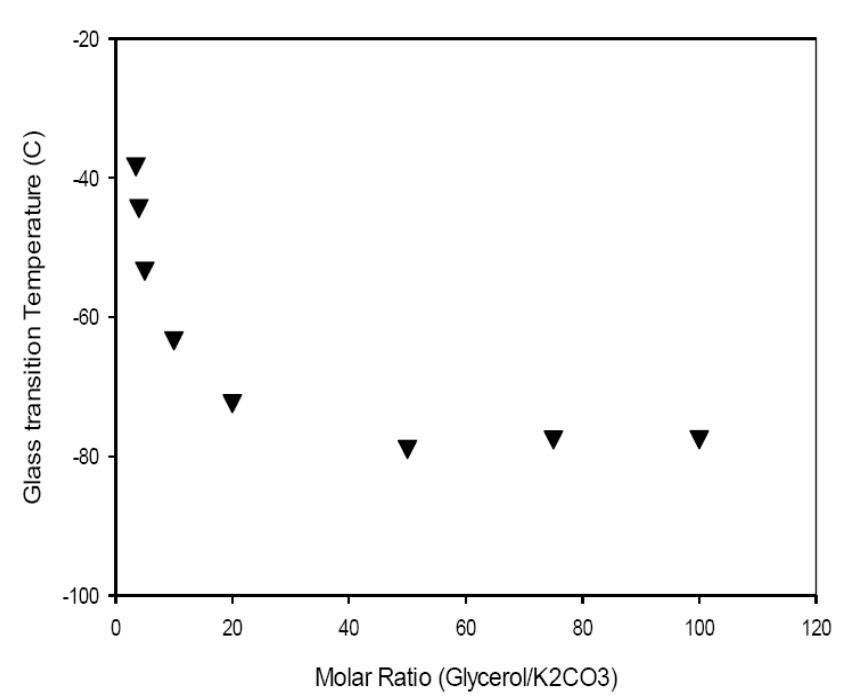

Fig. 1. Glass transition temperature observed for the DES ratios studied

The group called the mixture Low Transition Temperature Mixture (LTTM). Apparently this system of potassium carbonate-glycerol is behaving in a similar way. In the following sections the results of the physical properties investigated for DES5-DES7 are presented and discussed.

\section{A. Density}

Density is one of the important physical properties of solvents. Temperature has a significant effect on density of solvents in general. Fig. 2 shows the effect of temperature on density of the three selected DES ratios in this work.

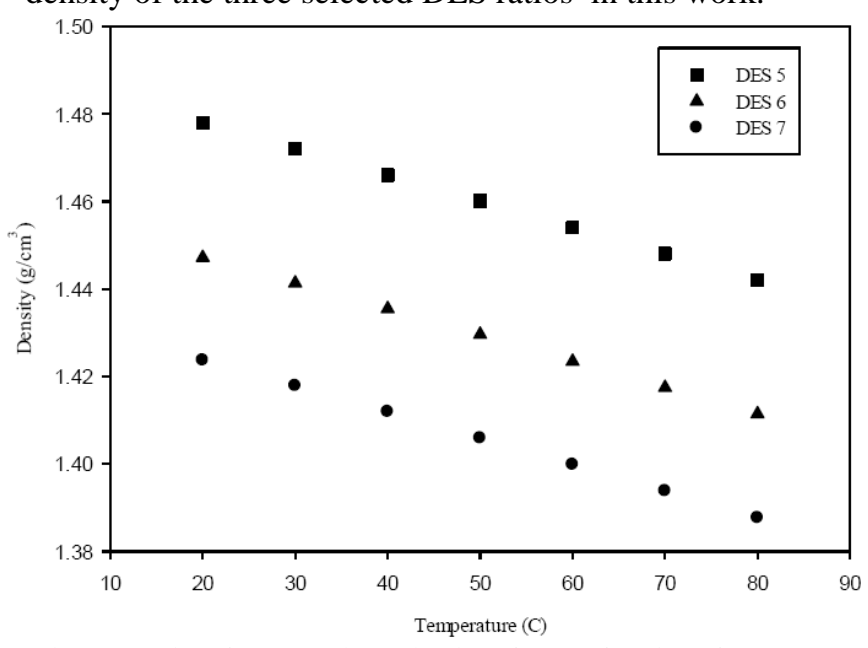

Fig. 2. Density of $\mathrm{K}_{2} \mathrm{CO}_{3}$ Glycerol ratios of 4-6 as function of temperature

It is clear from the figure that as the glycerol composition in the mixture increases the density of the DES decreases. This is expected and can be explained by the fact that the density of Glycerol $\left(1.291 \mathrm{~g} / \mathrm{cm}^{3}\right)$ is less than the density of $\mathrm{K}_{2} \mathrm{CO}_{3}\left(2.29 \mathrm{~g} / \mathrm{cm}^{3}\right)$. As the figure shows, the effect of temperature on the DES density is very clear as expected. The density of the DES decreased, as the temperature increased for all the three DESs. The decrease of the density of the DES as the temperature increase is explained by the increase in the mobility of the DES molecules and the thermal expansion of the DES volume as a result on the temperature increase. The relationship between the density and temperature is almost perfectly linear with an $\mathrm{R}^{2}$ value of more than 0.999 for all the three DESs.

\section{B. Viscosity}

The literature reports very limited experimental ILs viscosity data as function of temperature [5]. Studies conducted on DESs are even very scarce.

It is very important to establish a reliable viscosity database, due to the extreme importance of this physical property for equipment design and fluid flow calculations. By having such data at the design stage, the considered DES system can be synthesized to achieve the least value for viscosity. In addition, by knowing the temperature effect on viscosity, energy requirements for processing these fluids can be reduced [18] In general, ILs have exponentially decaying viscosity-temperature profiles [19]. Similar behavior has also been reported for some DES systems [5].

As expected, viscosity of this DES decreased with increasing temperature as shown in Fig. 3.

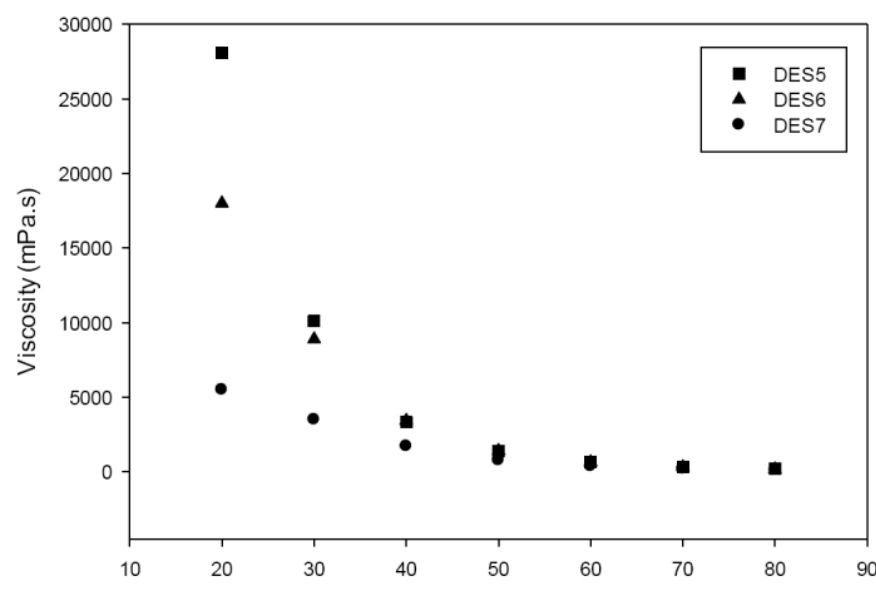

Fig. 3. Dynamic viscosity of selected DESs as function of temperature

At low temperatures the difference in the viscosities of the three ratios was very clear and DES5 had the highest viscosity among the three. As the temperature increased the difference in viscosity of the three ratios decreased and they almost had the same viscosity at temperatures $50^{\circ} \mathrm{C}-80^{\circ} \mathrm{C}$. This indicates that for applications requiring low solution viscosities (like reactions and 1-1 extraction applications) DES6 and DES7 are recommended (when used at room temperature). To use DES5 the mid-range temperature (40 and above) should be used.

The relationship between the salt to HBD molar ratio and viscosity for the studied DESs is also clear from the results attained. As the molar amount of HBD in the DES mixture increases, the viscosity values are dampened. But this effect diminishes at very high HBD compositions. Comparing these readings to the viscosity of pure glycerol at different temperatures [20], indicates similar trend of variation. Adding more glycerol to the DESs produces a composite viscosity closer to pure glycerol, whereas adding more salt in the mixture increases the viscosity considerably.

\section{Surface Tension}

Surface tension is one of the very important physical properties that determine the suitability of a given liquid in mass transfer processes such as emulsification or separation [21]-[23]. There is limited information on the surface tension of Deep Eutectic Solvents in literature. Fig. 4 shows the variation of the surface tension of the three DESs ratios with temperature. 


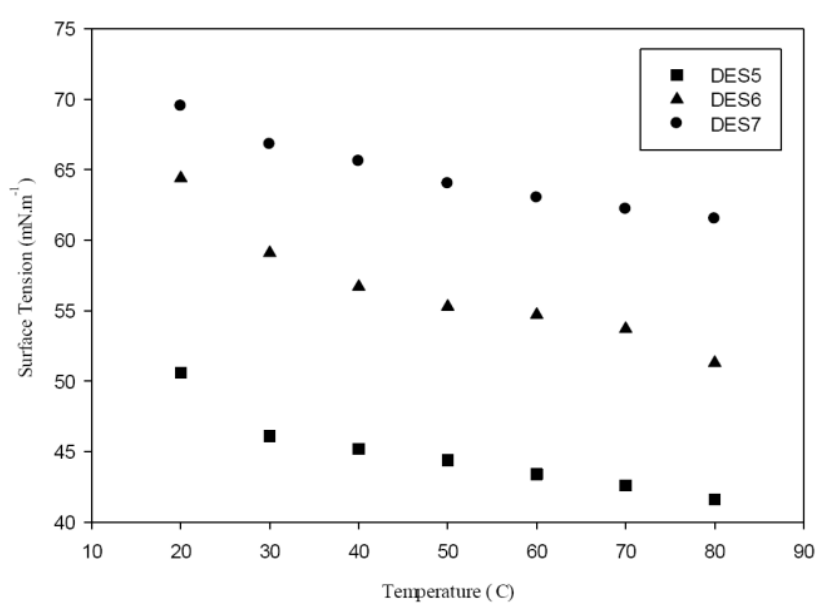

Fig. 4 Surface tension of selected DES ratios as function of temperature

For DES7, at room temperature, the surface tension value was $69 \mathrm{mN} / \mathrm{m}$. It decreased with increase in temperature to 63 $\mathrm{mN} / \mathrm{m}$ at $75{ }^{\circ} \mathrm{C}$. Similar trends were observed for DES5 and DES6. For DES5, the values obtained were from 51 to 42 in the temperature range. These are typical values for glycerol-based DESs [16]. As the temperature increases, the kinetic energy of the molecule increases. Thus, the attractive forces are weakened. The rate of decrease of surface tension with increase in temperature was found to be grater at higher ratios. Increase in the amount of $\mathrm{K}_{2} \mathrm{CO}_{3}$ led to decrease in the surface tension. This implies that the dissociated cations and anions occupy the interstitial space between the glycerol molecules and have weakened the attractive forces on the surface. This is important for potential applications where the properties of the DESs could be modified for given conditions.

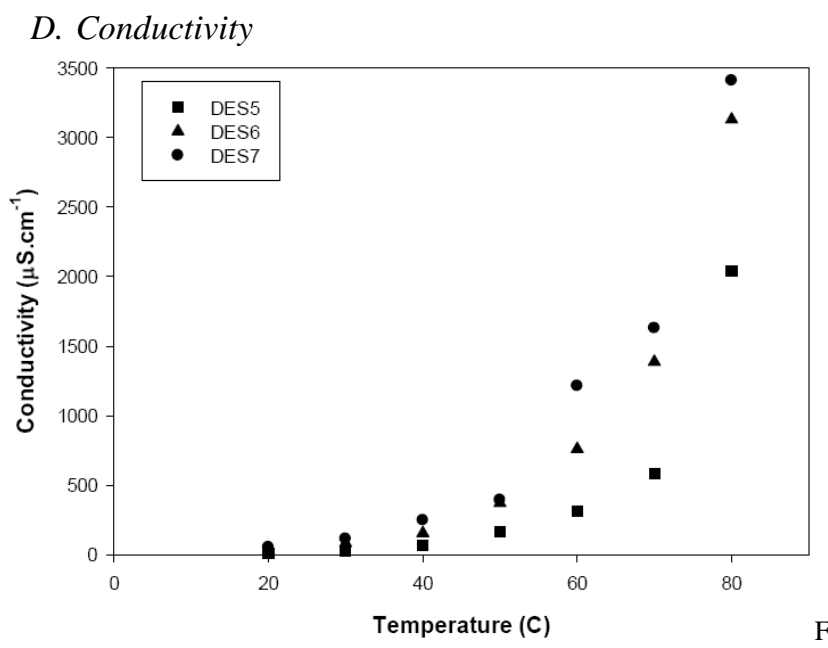

5. Conductivity of selected DESs as function of temperature.

Owing to their relatively high viscosities most of DESs exhibit poor ionic conductivity [24] and the DES in this work is no exception. Fig. 5 shows the conductivity of the three DESs in $\mu S / \mathrm{cm}$ as function of temperature.

The maximum conductivity of the DESs was about 3.5 $\mathrm{mS} / \mathrm{cm}$ for DES7 at $80^{\circ} \mathrm{C}$. The conductivity for all DESs was very small $(7.89 \mu \mathrm{S} / \mathrm{cm})$ at $20{ }^{\circ} \mathrm{C}$. The effect of temperature on the conductivity is very clear for the three DESs. As the temperature increased the conductivity increased significantly. The data showed a clear trend of increased conductivity of the DESs as the ratio of Glycerol: $\mathrm{K}_{2} \mathrm{CO}_{3}$ increased. The data also showed that the difference in the conductivities between the three DESs was more clear at high temperatures than at low temperatures at which the conductivity was almost the same for the three DESs.

\section{E. Refractive Index (RI)}

Refractive Index is another important DES property. It is the ratio of the speed of light in vacuum relative to that in a given sample. The index depends directly on electrical permittivity and magnetic permeability of the material. Thus, it is used to determine sample purity or its concentration in a given matrix. Fig. 6 shows the values of refractive index of the DESs as function of temperature.

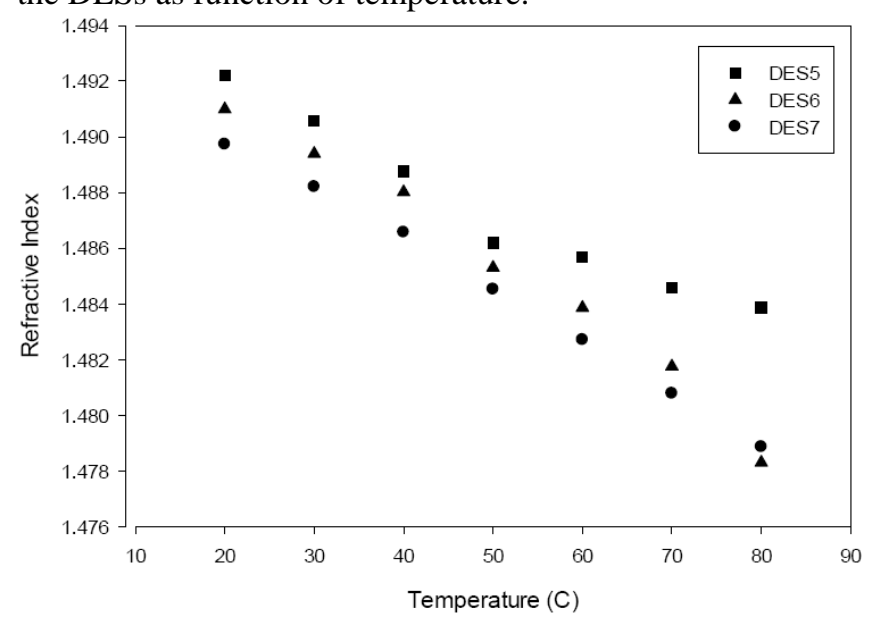

Fig. 6. Refractive indices for selected DESs as function of temperature

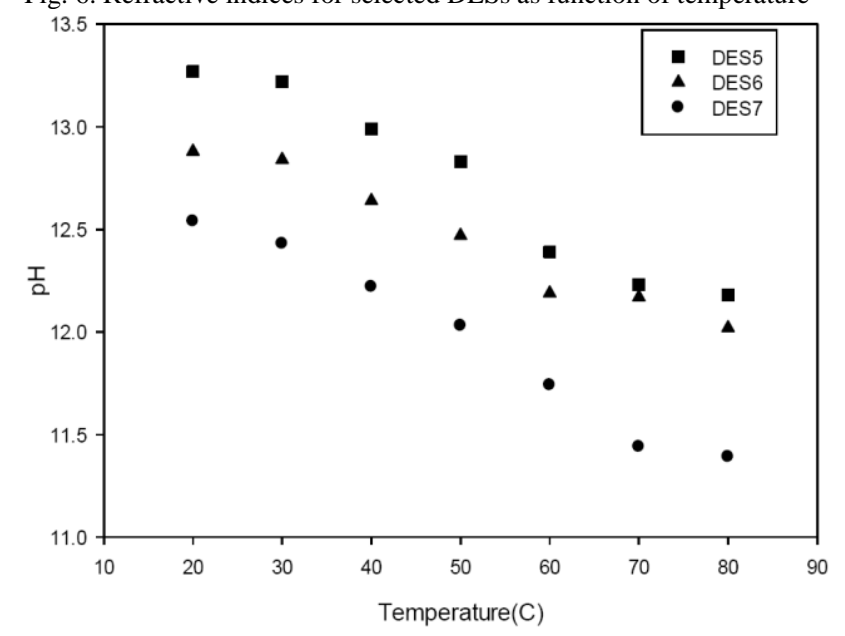

Fig. 7. $\mathrm{pH}$ for selected DESs as function of temperature

The values of RI decreased with the increase in temperature. For DES5, the values changed linearly (1.492 to 1.484) with temperature. DES6 and DES7 exhibited similar trends, but wider ranges and higher slopes.

\section{F. $p H$}

The $\mathrm{pH}$ is important in applications related to catalytic reactions, bioreactions, pharmacuitical preparations and in corrosion studies. In this work, the $\mathrm{pH}$ values of the DESs varied with temperature in the range of 11.5-13.5. In general the studied DESs were basic with decreasing basicity with temperature. Fig. 7 shows $\mathrm{pH}$ of the DESs as function of temperature.

As shown in Fig. 7. the pH of DES5 attained the highest values for all temperatures. Other DES ratios exhibited similar behavior with temperature.

Generally, the acidity or basicity of ionic liquids is governed by the strength of the cation, the anion, or by the combination of the cation and anion. Conventionally, basic 
ionic liquids are synthesized by either selecting a basic anion (lactate, formate, carboxylates and the dicyanamide) or by incorporating a basic site into the cation. For the case of DES, the nature of the salt and HBD dictates the basicity of the mixture. Similar to the successful use of basic ionic liquids in variety of catalyzed reactions such as the Michael addition, Heck reaction and Markovnikov addition [25], the newly developed DES has the potential for use in such applications.

\section{CONCLUSION}

In this work potassium carbonate has been used as the salt ingredient in an attempt to prepare a DES with glycerol as a hydrogen bond donor. The system didn't show freezing points, instead glass transition temperatures were observed. The physical properties including density, viscosity, surface tension, refractive index, conductivity and $\mathrm{pH}$ were measured and reported as a function of temperature for three different molar ratios of salt to HBD. It was found that the molar composition of this system has a pronounced effect on the physical properties.

Because of its high viscosity, density and surface tension at room temperature, this system need to be used at relatively moderate temperatures to improve its fluidity and transport properties. The studied DESs ratios $\mathrm{pH}$ values were basic with little decreasing basicity with temperature increase. This makes it suitable for certain applications than involve basic media. The refractive index and surface tension values for this system are strong function of salt: HBD molar ratio and temperature. This important behavior can be used to tune the composition and temperature to be in favor of the application sought. The effect of temperature is also manifested on the value of conductivity. All studied molar ratios have close conductivities at low temperatures; on the other hand, these ratios attain considerably large differences in conductivity at higher temperatures. This draws the attention of the importance these new electrolytes may bring to the electrochemical applications.

The current investigation opens the door to more thorough investigation of the chemical and mechanical applications of these new fluid. Further studies are needed to cover possible application of these DESs as solvents, reaction media, catalysts, geometry directing agents and electrolytes.

\section{ACKNOWLEDGMENTS}

This research was funded by the Department of Petroleum and Chemical Engineering, Sultan Qaboos University, Sultanate of Oman.

\section{REFERENCES}

[1] M. J. Earle, J. M. Esperanca, M. A. Gilea, J. N. Conogia, L. P. N. Rebelo, J. W. Magee, K. R. Seddon, and J. A. Widegren, "The distillation and volatility of ionic liquids," Nature Letters, vol. 439, pp. 831-834, 2006.

[2] J. M. S. S. Esperanca, J. N. Canongia Lopes, M. Tariq, L. M. N. B. F. Santos, J. W. Magee, and L. P. N. Rebelo, "Volatility of Aprotic Ionic Liquids - A Review," Journal of Chemical Engineering Data, vol. 55, pp. 2-12, 2010.

[3] M. J. Earle and K. R. Seddon, "Ionic liquids: Green solvents for the future," Pure Appl. Chem., vol. 72, pp. 1391-1398, 2000.

[4] P. Wasserscheid and T. Welton, Ionic Liquids in Synthesis, Weinheim: Wiley-VCH, 2003.

[5] M. A. Kareem, F. S. Mjalli, M. A. Hashim, and I. M. AlNashef, "Phosphonium-Based Ionic Liquids Analogues and Their Physical Properties," J. Chem. Eng. Data, vol. 55, p. 4632-4637, 2010.
[6] J. Sun, M. Forsyth, and D. R. MacFarlane, "Room-temperature molten salts based on the quaternary ammonium ion," J. Phys. Chem. B., pp. 8858-8864, 1998.

[7] M. Hayyan, F. S. Mjalli, A. M. Hashim, and I. M. AlNashef, "A novel technique for separating glycerine from palm oil-based biodiesel using ionic liquids," Fuel Processing Technology, vol. 91, pp. 116-120, 2010.

[8] S. Zhang, Y. Chen, R. X. F. Ren, Y. Zhang, and J. Zha, "Solubility of $\mathrm{CO} 2$ in sulfonate ionic liquids at high pressure," J. Chem. Eng. Data, vol. 50 , pp. 230-233, 2005 .

[9] A. P. Abbott, G. Capper, K. J. McKenzie, and K. S. Ryder, "Voltammetric and impedance studies of the electropolishing of type 316 stainless steel in a choline chloride based ionic liquid," Electrochim. Acta, vol. 51, p. 4420, 2006.

[10] A. P. Abbott, D. L. Davies, G. Capper, R. K. Rasheed, and T. Vasuki, "Ionic liquids and their use as solvents," US Patent US7183433, 2007.

[11] A. P. Abbott, J. C. Barron, K. S. Ryder and D. Wilson, "Eutectic-Based Ionic Liquids with Metal-Containing Anions and Cations," Chem. Eur. J. , vol. 13, pp. 6495-6501, 2007.

[12] J. Leonard, B. Lygo, and G. Procter, Advanced Practical Organic Chemistry, Stanley Thomas Publishers Ltd., 1998.

[13] J. T. Cullinane, Thermodynamics and Kinetics of Aqueous Piperazine with Potassium Carbonate for Carbon Dioxide Absorption, 2005.

[14] M. Pagliaro and M. Rossi, The Future of Glycerol, 2nd ed., RSC Publishing, 2010.

[15] A. Qamar and B. Leo, "Synthesis and characterization of choline chloride based binary mixtures," ECS Transactions, vol. 33, no. 7, pp. 49-59, 2010.

[16] R. C. Harris, "Physical Properties of Alcohol Based Deep Eutectic Solvents," PhD Thesis, University of Leicester, 2008.

[17] M. Francisco, A. Bruinhorst, L. Zubeir, C. Peters, and M. Kroon, "A new low transition temperature mixture (LTTM) formed by choline chloride + lactic acid: Characterization as solvent for CO2 capture," Fluid Phase Equilibria vol. 340 pp. 77-84, 2013.

[18] A. Hayyan, F. S. Mjalli, I. M. AlNashef, T. Al-Wahaibi, Y. Al-Wahaibi and M. A. Hashim, "Fruit sugar-based deep eutectic solvents and their physical properties," Thermochimica Acta, vol. 541, no. 10, pp. 70-75, 2012.

[19] M. H. Ghatee, M. Zare, and F. Moo, “Temperature-Dependent Density and Viscosity of the Ionic Liquids 1-Alkyl-3-methylimidazolium Iodides: Experiment and Molecular Dynamics Simulation," J. Chem. Eng. Data, vol. 55, no. 9, pp. 3084-3088, 2010.

[20] B. J. Segur and H. Oberstar, Ind. \& Eng. Chem., vol. 43, pp. 2117-2120, 1951.

[21] E. Jimenez, M. Cabanas, L. Segade, S. G. Garabal and H. Cases, Fluid Phase Equilib., vol. 180, pp. 151-164, 2001.

[22] B. L. Rhoda and L. Meng-Hui, Thermochimica Acta, in press, 2012.

[23] M. Zaira, L. Walter and D. D. M. Pablo, "Practical separation of alcohol-ester mixtures using Deep-Eutectic-Solvents," Tetrahedron Letters, in press , 2012.

[24] Q. Zhang, K. D. Vigier, S. Royer, and F. Jerome, "Deep eutectic solvents: syntheses, properties and applications," Chem. Soc. Rev., vol. 41, pp. 7108-7146, 2012.

[25] X. Lin-Fei, Y. Qun-Feng, X. Chun-Gu, and X. Li-Wen, "Supported basic ionic liquid: Highly effective catalyst for the synthesis of 1,2-propylene glycol from hydrolysis of propylene carbonate," Journal of Molecular Catalysis A: Chemical, vol. 279, pp. 230-234, 2008.

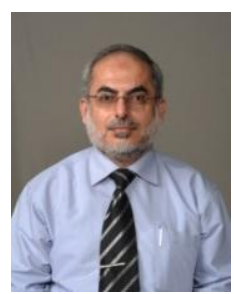

Jamil Naser was born in Jordan in 1965. He obtained his B.Sc. in chemical engineering from Jordan University of Science and Technology, Irbid, Jordan in 1989. M.Sc. in chemical engineering from the University of Jordan, Amman, Jordan in 1993. PhD in chemical engineering from University of Alabama in Huntsville, Huntsville, Alabama in 1998

He worked as a research associate at the Department of Chemical and Materials Engineering, University of Alabama in Huntsville in 1999. Adjunct faculty member at Alabama A\& M University in 2000. Assistant Professor at the Department of Chemical Engineering, Tuskegee University, Tuskegee, Alabama from 2001-2008 and currently he is an assistant professor at the Department of Petroleum and Chemical Engineering at Sultan Qaboos University, Muscat, Oman since 2008. His research experience includes Liquid Phase Sintering in Microgravity, Industrial wastewater treatment by floatation, adsorption for water and wastewater treatment, and recently Deep Eutectic Solvents properties and applications.

Dr. Naser is a member of the American Institute of Chemical Engineers and the Jordanian Engineers Association. 